Z. klin. Chem. u. klin. Biochem.

7. Jg., S. $356-360$, Juli 1969

\title{
Vollautomatische enzymatische Bestimmung des freien Glycerins im Blut mit dem AutoAnalyzer
}

\author{
Von U. Harding unter Mitarbeit von G. HeINZEL \\ Aus den Biocbemilscben Forscbungslaboratorien der Firma Dr. Karl Thomae GmbH, Biberacb|Riß
}

(Eingegangen am 7. März 1969)

\begin{abstract}
Es wird ein Verfahren zur vollautomatischen Bestimmung des freien Glycerins im Vollblut mit dem Teçnicon AutoAnalyzer beschrieben. Es beruht auf der von O. Wreland angegebenen enzymatischen Methode. Unter Verwendung von $100 \mu l$ - bzw. $200 \mu l$-Blutproben können 60 Bestimmungen in der Stunde durchgeführt werden. Dabei werden mindestens noch 0,2 bzw. $0,1 \mathrm{mg} / 100 \mathrm{~m} / \mathrm{Glycerin}$ einwandfrei erfaßt. Andere Blutbestandteile stören nicht. Meßgenaưigkeit, Wiederfindungsrate und Vergleich mit der manuellen Methode werden angegeben.
\end{abstract}

\section{The fully automated determination of free glycerol in blood with the Auto Analyser}

A fully automated method is described for the measurement of free glycerol in whole blood with the Technicon AutoAnalyzer. It is based on the enzymic determination of glycerol according to $O$. WIELAND. Using $100 \mu \mathrm{l}$ or $200 \mu /$ blood samples, 60 determinations per hour are possible. Values as low as 0.2 or $0.1 \mathrm{mg} / 100 \mathrm{~m} /$ glycerol respectively may be easily measured. Other blood components do not interfer. The accuracy, the recovery and a comparison with the manual method are given.

$\mathrm{Zu}$ den wichtigsten Metaboliten des Fettstoffwechsels gehört neben freien Fettsäuren, Triglyceriden und Ketonkörpern auch das freie Glycerin, ein Mobilisationsprodukt der Lipolyse (1). Bei in vitro-Versuchen ist das abgegebene Glycerin ein zuverlässiges Maß für die Lipolyserate (2).

Zur Messung des Glycerins in Blut oder Inkubationsmedien werden empfindliche, spezifische und leicht $\mathrm{zu}$ handhabende Methoden benötigt. Die bekannten photometrischen und fluorimetrischen Verfahren (3-11) sind meist zu wenig spezifisch. Dagegen sind gute enzymatische Bestimmungsmethoden bekannt (12-19). Die Bewältigung einer großen Zahl anfallender Proben erfordert weitgehende Automatisierung. Für das System des Technicon AutoAnalyzers ist bisher jedoch noch keine Möglichkeit zur Messung der geringen Blutkonzentrationen an Glycerin (Normalbereich bei Ratten nach unseren Messungen 0,5-4,0 mg/100 $\mathrm{ml}$ je nach Dauer der Nahrungskarenz) bekannt. Es liegen lediglich semiautomatische Verfahren zur Bestimmung der Triglyceride vor, in deren Endphase Glycerin fluorimetrisch (20) oder kolorimetrisch (21) gemessen wird. Eine enzymatische Methode nach ANronis (22) wird auch nur für Glycerid-Glycerin angegeben.

Wir möchten im folgenden eine vollautomatische Bestimmung des freien Glycerins aus Vollblut am AutoAnalyzer vorschlagen, die auf der Basis der Methode von O. WIELANi (12) ausgearbeitet wurde und sehr einfach durchführbar ist.

\section{Methodik}

Prinzip

Die zu messenden Blutproben werden mit $\mathrm{NaCl}-\mathrm{Lösung}$ verdünnt in den AutoAnalyzer gegeben und durch Dialyse gegen $\mathrm{NaCl}$ enteiweißt, wobei $50 \%$ des Glycerins in die zur Messung gehende $\mathrm{NaCl}$-Lösung diffundiert. Das Dialysat wird dann mit
Reaktionsgemisçh verẹinigt, wobei folgende U̇msetzungen stattfinden ${ }^{1}$ ):

$$
\begin{aligned}
& \text { ATP + Glycerin } \stackrel{\text { GK }}{\longrightarrow} \alpha \text {-Glycerophosphat + ADP; } \\
& \alpha \text {-Glycerophosphat } \stackrel{\text { GDH }}{\longrightarrow} \text { Dihydroxyacetonphosphat- } \\
& +\mathrm{NAD}+\text { Hydrazin } \quad \text { Hydrazon }+\mathrm{NADH}_{2} \text {. }
\end{aligned}
$$

Das $\mathrm{NADH}_{2}$ ist der phosphorylierten Glycerinmenge äquimolar und wird fluorimetrisch gemessen. Die Reduktion von Dihydroxyacetonphosphat wird durch das Hydrazin verhindert.

Reagenzien und Lösungen

Reagenzien

$\mathrm{NaCl}$ p. a. (Merck, 6406)

Tween 20 (Schuchardt, TW 125)

L-Glycin (Merck, 4200)

Hydrazinhydrat (Merck 4604)

$\mathrm{KOH}$ p. a. (Merck 5033)

$\mathrm{MgCl}_{2} \cdot 6 \mathrm{H}_{2} \mathrm{O}$ p. a. (Merck 5833)

ATP (Boehringer Mannheim, 15028 NAAH)

NAD (Boehringer Mannheim, 15300 CNAA)

GK (Boehringer Mannheim, 15746 EGAP)

GDH (Boehringer Mannheim, 15.135 EGAQ)

Glycerin p. a. (Merck, 4094)

\section{Verdünnungslösung}

0,9proz. NaCl-Lösung $+0,5 \mathrm{~m} / l$ Tween 20. Pro Liter wird eine kleine Spatelspitze Phenol zugegeben.

\section{Reaktionsgemisch}

Diese Lösung muß unbedingt täglich neu angesetzt werden. Es empfiehlt sich, zunächst zu einem aliquoten Teil die beiden Enzyme zuzugeben, den beschriebenen Vorversuch zu machen und dann erst die Gesamtlösung mit den Enzymen zu vereinigen.

$9 \mathrm{~g}$ Glycin $+124,8 \mathrm{~g}$ Hydrazinhydrat mit $1 \mathrm{~N} \mathrm{KOH}$ auf $\mathrm{pH} 9,8$ einstellen, danach $240 \mathrm{mg} \mathrm{MgCl}_{2} \cdot 6 \mathrm{H}_{2} \mathrm{O}, 500 \mathrm{mg}$ ATP und $225 \mathrm{mg}$ NAD zugeben und mit bidest. Wasser auf $600 \mathrm{ml}$ auffüllen. Anschließend mit $20 \mathrm{mg} \mathrm{GDH}, 2 \mathrm{mg} \mathrm{GK}$ und einigen Tropfen Tween 20 versetzen und gut schütteln.

2) Die Abkürzung GK (Glycerokinase) wird hier gebraucht für ATP: Glycerin Phosphotransferaso (EC 2.7.1.30) und GDH (Glycerinphosphatdehydrogenase) für I-Glycerin-3-phosphat: NAD Oxydoreduktase (EC 1.1.1.8). 


\section{Glycerinstandards}

Einwiegen von Glycerin und Auffüllen mit bidest. Wasser in folgenden Konzentrationen: 0,5, 1, 1,5, 2, 3, 5, $10 \mathrm{mg} / 100 \mathrm{~m} /$. Auch hier wird pro Liter eine kleine Spatelspitze Phenol zugesetzt. Die Lösungen sind im Kühlschrank 4 Wochen unverändert haltbar.

\section{Gcräte}

Als Meßgerät dient der Technicon AutoAnalyzer (Standardausführung) mit Probennehmer II (Nockenscheibe 1:1 für 60 Proben pro Std.), Proportionierpumpe, Dialysator $\left(37^{\circ}\right)$, Doppelheizbad, Tygon-Schläuchen, Durchflußfluorimeter und Linienschreiber. Anregungsfilter $360 \mathrm{~nm}$ (Technicon), Emmissionsfilter $460 \mathrm{~nm}$ (Schott und Gen., Mainz). Probenbecher aus Glas oder Polyäthylen, da Polystyrenbecher von Technicon nicht geeignet sind. Gut bewährt haben sich bei uns auch Probenbecher der Firma AS-Nunc (Algade 8, Roskilde, Dänemark).

Verdünnung der Blutproben erfolgt mit Citopipetten der Fa. E. Bühler, Tübingen, die Durchmischung mit Rührstab (3350) der Firma Eppendorf Gerätebau.

Der Vorversuch und die zum Vergleich herangezogene manuelle Bestimmung des Glycerins nach WreLAND (12) wurde an einem Eppendorf-Photometer (1101 M), Filter $334 \mathrm{~nm}$, mit Kompensationsschreiber (4412) und Trafostufe (1853), die auf den Registrierbereich $0-0,25$ eingestellt war, mit Halbmikro-Quarzküvetten ( $20 \mathrm{~mm}$ Lichtweg) bzw. mit $10 \mathrm{~mm}$ Quarzküvetten beim Vorversuch, ausgeführt.

\section{Ausführung}

Aufbau und Arbeitsweise der AutoAnalyzer-Anlage ergeben sich aus Abbildung 1. Zur Inbetriebnahme wird die Apparatur zu-

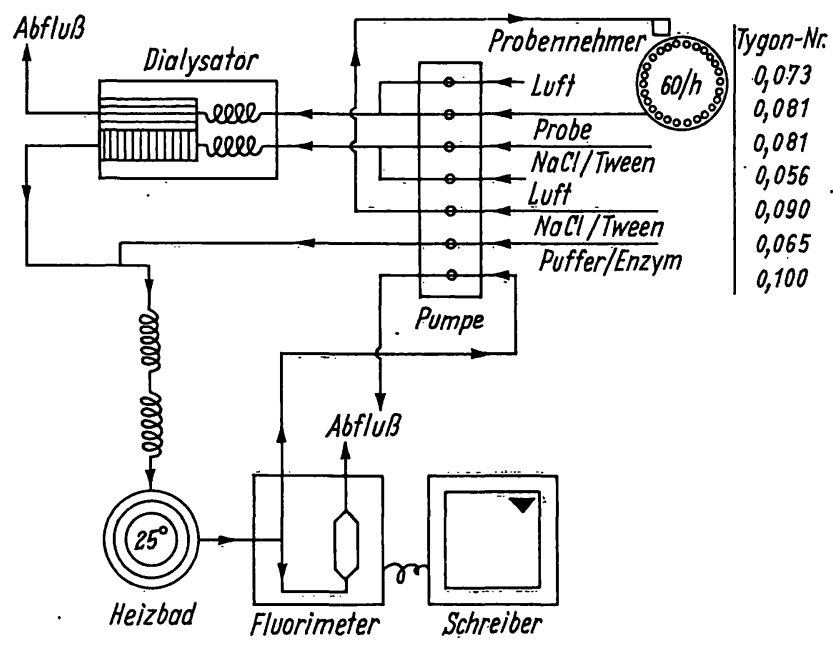

Abb. 1

Blut-Glycerin-Bestimmung im AutoAnalyzer, Fließ-Schema. Die Weiten der Tygonschläuche in der Proportionierpumpe sind nach dem Technicon-Datenblatt für Schlauchgrößen angegeben

nächst 30 Min. nur mit der Verdünnungslösung durchspült und weitere $30 \mathrm{Min}$. mit Verdünnungs- und Reaktionsgemisch. Danach wird die Basislinie eingestellt (Schreiberausschlag 3-5\%), deren Verlauf ërst nach dieser Zeit konstant wird.

\section{Vorversuch}

Daneben führen wir stets einen einfachen Vorversuch durch, mit dem die Brauchbarkeit der Reaktionslösung und der ausreichende Gehalt an Enzymen kontrolliert wird. $3,2 \mathrm{ml}$ des Reaktionsgemisches ohne Enzyme werden mit $5 \mathrm{~m} /$ Verdünnungslösung versetzt. $2 \mathrm{~m} /$ dieser Mischung werden mit $100 \mu /$ Glycerin $(2 \mathrm{mg} /$ $100 \mathrm{~m} /$ ) und $25 \mu /$ GDH vereinigt. Nach Eintritt einer konstanten Nulleinstellung am Eppendorf-Photometer wird die Reaktion mit $5 \mu /$ GK gestartet. Läuft die Reaktion ordnungsgemä $B$ ab, ergibt sich der Kurvenverlauf A der Abbildung 2. Ist die Enzymmenge zu gering oder das Reaktionsgemisch unbrauchbar (In-

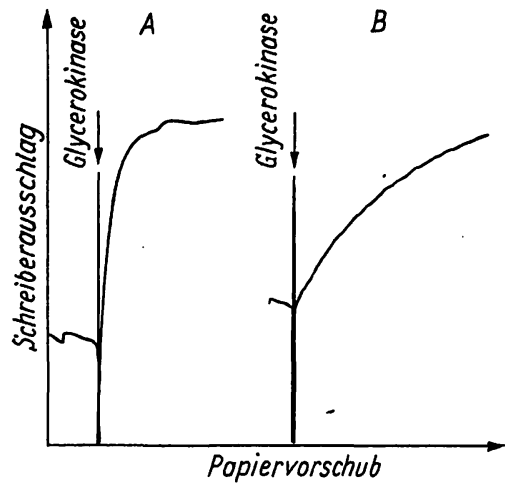

Abb. 2

Vorversuche zur Prüfung der Brauchbarkeit des Reaktionsgemisches für die automatische Glycerinbestimmung. Eppendorf-Photometer Papiervorschub des Schreibers $3 \mathrm{~cm} / \mathrm{Min}$. Geeignetes (A) und ungeeignetes Reaktionsgemisch (B)

stabilität und Sauberkeit des Hydrazins, pH), dagegen der Kurvenverlauf $\mathrm{B}$ oder gar keine Reaktion. In diesem Fall werden nochmals $10 \mu / \mathrm{GDH}$ (entsprechend $3,5 \mathrm{ml}$ auf $600 \mathrm{~m} /$ Lösung) zugesetzt. Bringt dies keine Verbesserung, so ist das Reaktionsgemisch neu anzusetzen.

\section{Durchfiubrung der Bestimmung}

$100 \mu l$ des zu untersuchenden Blutes bzw. der Standardlösungen werden in den Probenbechern mit jeweils $1,25 \mathrm{~m} /$ Verdünnungslösung versetzt, gut gemischt und in den Probennehmer eingesetzt. Zu Beginn jeder Versuchsreihe werden je 2-3 Proben von 2 verschiedenen Standards ( 2 und $3 \mathrm{mg} / 100 \mathrm{ml}$ ) gemessen, wobei die Fluorimeterempfindlichkeit so eingestellt werden sollte, daß der Schreiberausschlag bei $2 \mathrm{mg} / 100 \mathrm{ml}$ etwa $40-50 \%$ beträgt. 2 weitere Standardproben werden nach je 40 Messungen bestimmt.

Die Entnahme am Probennehmer erfolgt mit einer Rate von 60 pro Std., wobei nach Transport durch die Pumpe mit Luft segmentiert wird. Beim Dialysieren $\left(37^{\circ}\right)$ gegen Verdünnungslösung (ebenfalls durch Luft segmentiert) gelangen $50 \%$ der Glycerinmenge in das weitere Analysensystem, wo sie mit dem Reaktionsgemisch vereinigt werden. Nach Durchgang durch 2 Doppelmixspiralen und den Thermostaten (Heizbad $25^{\circ}$ ) erfolgt Messung im Fluorimeter. Ausgewertet wird mit der Universalschablone durch Aufstellung einer Eichkurve.

Die im Experiment gewonnenen Blutproben müssen nicht sofort der Messung zugeleitet werden. Versuche bei Raumtemperatur und bei $2^{\circ}$ im Kühlschrank haben gezeigt, daß in jedem Fall die bis zu 5 Std. nach der Entnahme gemessenen Glycerinkonzentrationen gleich bleiben.

Die manuellen Vergleichsmessungen wurden nach der Vorschrift von O. Wieland (12) durchgeführt, vobei die Enzymkonzentrationen $2 \mathrm{mg} / 10 \mathrm{ml}$ bei $\mathrm{GDH}$ und $10 \mu / \mathrm{GK}$ pro Probe bctrugen. Die Umsatzrate wurde am Eppendorf-Schreiber nach Stillstand der Reaktion abgelesen.

\section{Charakteristik der Methode}

\section{Zur technischen Durchführung}

Um zu prüfen, ob bei der in Anwendung kommenden Dialyse gegen die gleiche Verdünnungslösung tatsä̀chlich ein konstanter Anteil an Glycerin gemessen wird, wurde mit Standardlösungen verschiedener Konzentrationen ein Umpolungsversuch gemacht. Dazu wurde der. Probenzulauf nach Abbildung $1 \mathrm{mit} \mathrm{dem}$ Zufluß von $\mathrm{NaCl}+$ Tween vertauscht und die aufgezeichnieten Meßwerte mit denen der normalen Dialyse verglichen. Tabelle 1 zeigt, daß bei den verwendeten Einwaage-Konzentrationen der Schreiberausschlag in 
Tab. 1

Bestimmung der Eliminierungsrate von Glycerin bei der Dialyse von Standardlösungen am AutoAnalyzer. Jede Angabe ist der Mittelwert aus 4 Messungen

\begin{tabular}{ccc}
\hline $\begin{array}{c}\text { eingewogene } \\
\text { Konzentration } \\
\text { des Standards } \\
{[\mathrm{mg} / 100 \mathrm{ml}]}\end{array}$ & $\begin{array}{c}\text { gemessene Glycerinkonzentration in } \\
\text { Skalenteilen am Schreiber } \\
\text { umgepolte Dialyse }\end{array}$ \\
\hline 1 & normale Dialyse & umgepols \\
2 & 30,5 & 15,0 \\
3 & 44,0 & 31,5 \\
4 & 58,5 & 44,0 \\
5 & 76,0 & 60,0 \\
& & 74,0 \\
\hline
\end{tabular}

beiden Fällen identisch ist, d. h. bei der Dialyse $50 \%$ des Glycerins der Analyse zugeführt werden und $50 \%$ verlorengehen.

Um die Eigenfluoreszenz der Proben zu messen, wurde Blut gegen folgende Lösungen dialysiert: a) Verdünnungslösung, b) Reaktionsgemisch ohne GDH, c) Reaktionsgemisch ohne GK. In keinem Fall trat eine

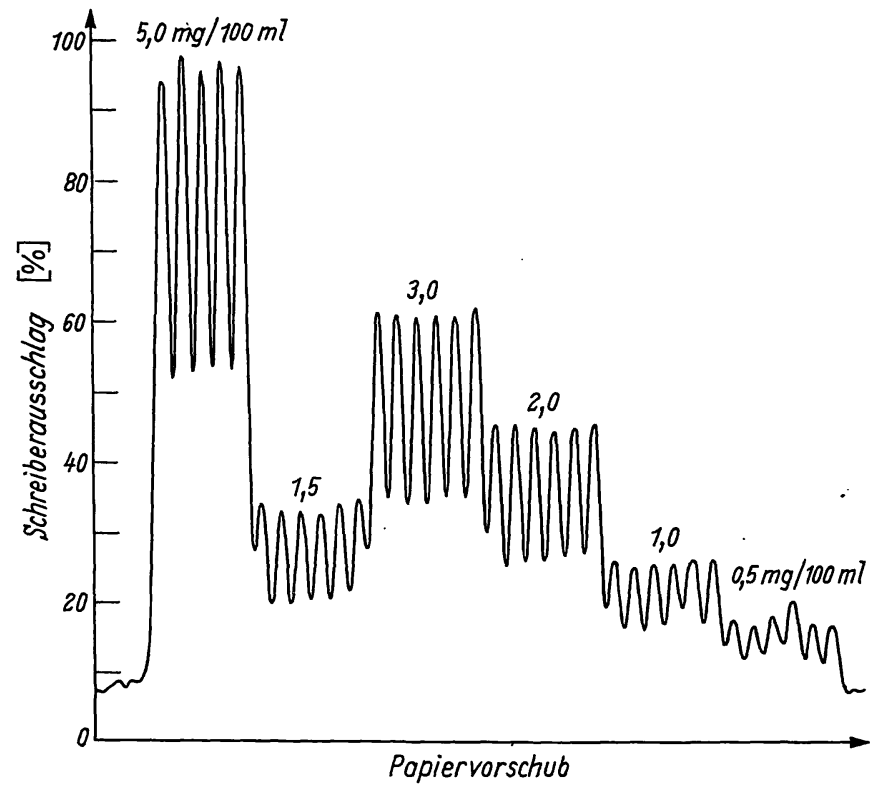

Abb. 3

AutoAnalyzer-Diagramm zum Nachweis der Beziehung zwischen Glycerinkonzentration und Fluoreszenz. Mehrfachmessung verschiedener Standardlösungen im Bereich von $0,5-5,0 \mathrm{mg} / 100 \mathrm{ml}$

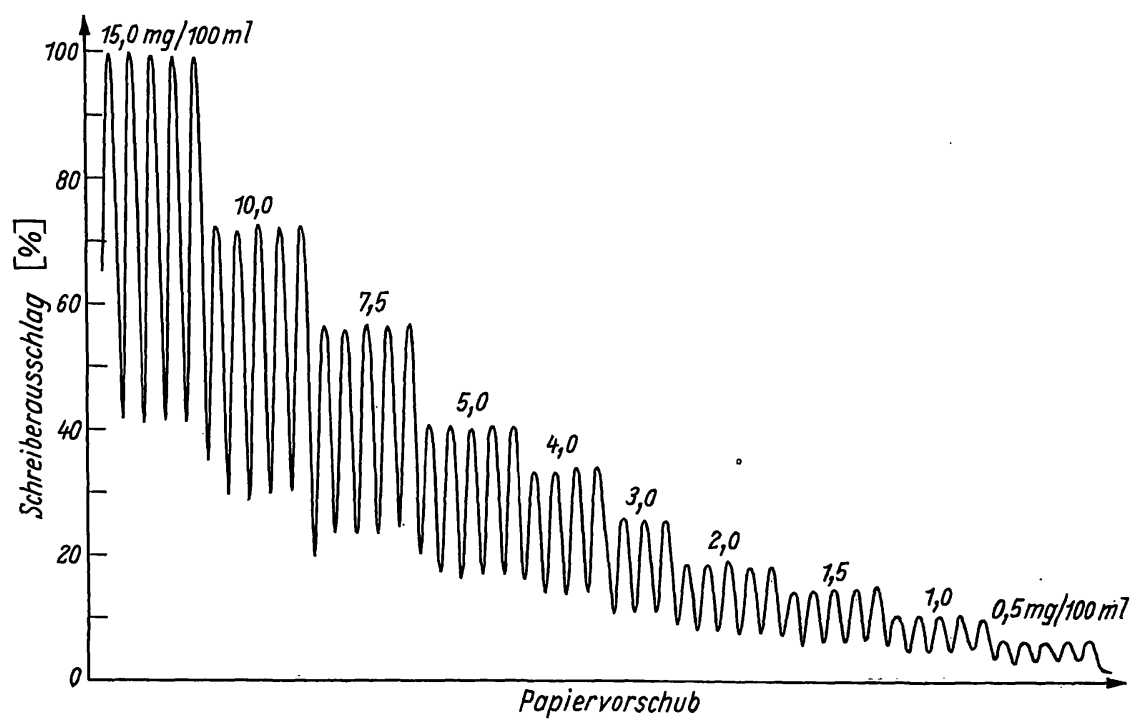

meßbare Fluoreszenz auf. Dies zeigt, daß die Bestimmung bei den angegebienen Wellenlängen durch andere Blutbestandteile nicht.gestört wird.

Unter den gewählten Bedingungen sind die Schreiberausschläge bei den für die Blutmessungen wichtigen Konzentrationen streng proportional der Glycerinmenge, wie es die Messung verschiedener Standards zeigt (Abb. 3).

\section{Präzision, Empfindliçhkeit \\ Meßgenauigkeit}

Zur Überprüfung der Meßgenauigkeit wurden Glycerinstandards (Einwaage-Konzentration $2 \mathrm{mg} / 100 \mathrm{~m} /$ bei Versuch 1-5 und $4 \mathrm{mg} / 100 \mathrm{ml}$ bei Versuch 6 , Tab. 2) zu verschiedenen Zeiten unter völlig gleichen Bedingungen gemessen. Von jeder Probe wurden 10 Meßwerte ermittelt und der mittlere Fehler des Mittelwertes $\left(S_{\bar{x}}\right)$ berechnet (Tab. 2). Er beträgt im Durchschnitt $0,01-0,02 \mathrm{mg} / 100 \mathrm{ml}$.

Tab. 2

Prüfung der Meßgenauigkeit der Glycerinbestimmung am AutoAnalyzer mit Hilfe von Standardlösungen. Angaben in $\mathrm{mg} / 100 \mathrm{ml}$ $\bar{x}=$ Mittelwert, $S \bar{x}=$ mittlerer Fehler des Mittelwertes. Versuch 1-5: Einwaage-Konz. $2 \mathrm{mg} / 100 \mathrm{ml}$, Versuch 6: Einwaage-Konz. $4 \mathrm{mg} / 100 \mathrm{ml}$

\begin{tabular}{|c|c|c|c|c|c|c|}
\hline Versuch & 1 & 2 & 3 & 4 & 5 & 6 \\
\hline \multirow[t]{10}{*}{$x$} & 1,94 & 1,98 & 2,03 & 2,00 & 1,95 & 4,00 \\
\hline & 1,90 & 2,00 & 2,05 & 1,95 & 1,95 & 4,05 \\
\hline & 1,96 & 2,01 & 2,02 & 2,03 & 2,00 & 3,90 \\
\hline & 1,96 & 2,00 & 2,02 & 2,03 & 1,95 & 3,90 \\
\hline & 1,98 & 1,96 & 2,00 & 2,00 & 2,00 & 4,10 \\
\hline & 2,02 & 1,98 & 2,05 & 2,00 & 1,95 & 4,00 \\
\hline & 2,00 & 2,00 & 2,06 & 2,07 & 1,95 & 4,05 \\
\hline & 2,00 & 2,02 & 2,00 & 2,00 & 1,95 & 3,95 \\
\hline & 2,00 & 2,00 & 2,00 & 2,00 & 2,00 & 4,00 \\
\hline & 2,00 & 2,00 & 2,08 & 2,03 & 2,00 & 4,05 \\
\hline$\overline{\mathbf{x}}$ & 1,98 & 2,00 & 2,03 & 2,01 & 1,97 & 4,00 \\
\hline $\mathrm{s}_{\overline{\mathrm{x}}}$ & 0,025 & 0,012 & 0,020 & 0,022 & 0,018 & 0,015 \\
\hline
\end{tabular}

Aus Abbildung 3 ist ersichtlich, daß bei Verwendung von $100 \mu l$-Proben der Schreiberausschlag bei $2 \mathrm{mg} /$ $100 \mathrm{~m} l$ von der Basislinie an gerechnet $40 \%$ beträgt.
Abb. 4

AutoAnalyzer-Diagramm zum Nachweis der Beziehung zwischen Glycerinkonzentration und Fluoreszenz. Mehrfachmessung verschiedener Standardlösungen im Beréich $0,5-15 \mathrm{mg} / 100 \mathrm{ml}$ 
Olnne Verschlechterung der Grundlinic ist es jedoch auch mïglich, den Ausschlng auf 50\% auszudehnen. Dic noch gut erfaßbaren Ausschläge von $5 \%$ bedeuten dann $0,2 \mathrm{mg} / 100 \mathrm{~m} /$ Glycerin, bei Verwendung von $200 \mu /$ Blut $0,1 \mathrm{mg} / 100 \mathrm{~m} /$ Glycerin als untere, melBbarc Konzentration. Nach $\Lambda$ bbildung 3 ist clsenfalls mit $100 \mu /-$ Prolsen ohne Verinderung der Fluorimetercinstellung und der Enzymmengen die obere Meßgrenze $5 \mathrm{mg} / 100 \mathrm{~m} /$, nach Neucinstellung des Meßgerïts (Redurierung dor Empfindlichkeit) $15 \mathrm{mg} / 100 \mathrm{ml}$ (Abl). 4).

\section{"IFicderfindungsialo}

$5 \mathrm{~m} /$ Rntteublut wurden mit $155 \mathrm{~m} /$ Verdümnungslösung verdüinnt und $1,25 \mathrm{~m} /$ dicser Mischung in Probenbechern entweder init $50 \mu /$ Verdünnungslösung oder $50 \mu l$ Glyeerinlösung verschiedener Kon\%entration, deren Gehalt zur Kontrolle ebenfalls mitgemessen wurde, versetzot ('I'ab. 3). Dic eingesetzten Glyecrinmengen wurden vollstündig wiedergefunden.

Tab. 3

Messung der Wloderflndungigrate bol Olycerlnbestlnmmungen am AutoAnalyzer durch Zugabe versehledener Mengen zu Blitproben. Die All-

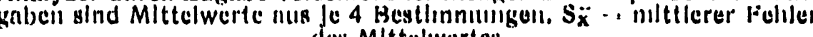
des Mittelwortes

\begin{tabular}{|c|c|c|c|}
\hline $\begin{array}{c}\text { Olycerlinkonze } \\
\text { Blutprobe }\end{array}$ & 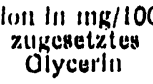 & $\begin{array}{l}\text { J. } \\
\text { Blint } \\
\text { alycerlin }\end{array}$ & $\begin{array}{l}\text { \% Wleder- } \\
\text { flindinis }\end{array}$ \\
\hline 2,00 : 0,07 : : & 0,80 . L 0,04 & $2,58 \neq 1.0,03$ & 102,5 \\
\hline $1,90 \quad 1: 0,05$ & $1,00 \pm 0,03$ & $2,00: 0,04$ & 100 \\
\hline $1,05: 1: 0,05$ & $1,90 \quad 1: 0,03$ & $3,00+0,05$ & 09 \\
\hline $1,80: 1: 0,03$ & $2,90 \pm 0,05$ & $4,70 \perp 0,08$ & 100 \\
\hline $1,05: \mathrm{L}: 0,05$ & 4,00 : 0,04 & $5,76: 1: 0,03$ & 97 \\
\hline $2,00: 5,05$ & 4,00 t. 0,04 & 6,80 i: 0,08 & 08,0 \\
\hline
\end{tabular}

\section{Vergleich quiscben mnnucller und anlomalischer Messung}

Es wurde ein Vergleich zwischen der AutoAnalyzcrmethode und der manucllen Bestimmung durchyeführt. Dayu wurde aus dem Serum von 8 Ratten (Probenmenge bei manucller Bestimmung $200 \mu \mathrm{l}$ ) der Glycuringehalt bestimmt ('Tab. 4). Dic durchschnitt-

Tab. 4

Vorglelch dor Meßslaten lin Rattenseriun bol dor emzymatlschen Olycerlinbestlinmung zwlsclien Hiandinethode und AutoAnalyzer. Dlo Aligaben slind Mittelworte in mig/100 inl mus o Messungen. S- - inltt-
lerer lochlor des Mittelwertes

\begin{tabular}{|c|c|c|c|}
\hline Tler & Alto Analyzor & Handinetliode & $\%$ Abwelchung \\
\hline 1 & $1,30+0,02$ & $1,20 \cdot 0,09$ & $+2,0$ \\
\hline 2 & $1,50: 1: 0,020$ & $1,00 \pm 0,09$ & 0 \\
\hline 3 & $1,21 \perp 0,04$ & $1,18 \perp 0,04$ & $+1,65$ \\
\hline 4 & $0,67 \pm 0,03$ & $0,64: 0,02$ & $+4,8$ \\
\hline E & $2,11+0,01$ & 2,17 :t 0,03 & $-2,8$ \\
\hline i & $1,58: 0,01$ & $1,00: 1: 0,03$ & $-5,1$ \\
\hline 7 & 1,74 d: 0,01 & $1,18: 2: 0,01$ & $-3,0$ \\
\hline 8 & $1,36: 0,01$ & $1,36: t: 0,01$ & 0 \\
\hline
\end{tabular}

liche Abwejeliung "der Mittelwerte liegt bei 2,6\%. Keine der beiden Methoden jedoch ergals in jedem Versuch höhere Wertc. Außcrdem stimmen dic AutoAnalyzerwerte besser überein (dic Endpusakteinstellung bei der Handmethode erfolgt zum Schluß schr nögernd und ist schlecht vom Reaktions-Drift zu unterscheiden). Deshalb beruhen dic Unterschicde wohl vornchmlich auf dem Meßfehler der Handbestimmung.

\section{Diskussion}

In dic chemischen Methoden \%ur Glyccrinbestimmung zwar empfindlich, aber oft unsperifisch und acitautwendig sind (23), versuchten wir \%wei hïufig verwendete enzymatische Verfahren auf den AutoAnalyzur \%u übertragen $(12,14)$.

Bei der Methode nach Kusu'z (14) muß vor "zugabe von GK der Reaktionsstillstand abgewartet werden. Da das AutoAnaly\%cr-System nur cine cinnalige Messung einer Probe gestattet, würde das Brgebnis auf diesse Weise durch unsperifische Reaktionen verfïlscht. 'Dics zeigte ein Versuch mit $200 \mu /$ Serum, das wir am AutoAnaly'zer dialysicrten und nach Vereinigung mit dem Reaktionsgcmisch mach KkLU'r's (14) ohne GK gcmessen haben. Wir fanden vorgetäuschte Glycerinwerte von $1-4 \mathrm{mg} / 100 \mathrm{~m} /$.

Bei der Methode nach WJulanND (12) dagegen ist das cntstchende NADH- ${ }_{2}$ der Glyccrinmenge direkt proportional, da im Blut $\alpha$-Glycurophosphat nicht vorhanden ist (12). Nllerdings ist hier des ungünstigen Renktionsgleichgewichts wegen Hyclrazin nötig, das jedoch das Reaktionsgemisch instabil macht. Wir kontrollicren dies daher bei jedem $\Lambda$ nsat: mit dem einfach zu handhabenden Vorversuch. Weiter wirkt sich die je nach Cilyecrinkon\%entration unterschiedliche Renktions\%cit bis \%ur Endpunktcinstellung bei der manuellen Messung nachteilig aus. Bei den sehr kleinen

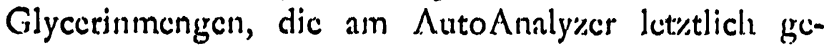
messen werden, genügen jedoch dic zur Verfügung stehenden Verweil\%eiten. Durch die Dialyse fallen dic dic Fluoreszenzanessung störcnden Bestandtcile des Blutes weg and die Glycerinmenge wird um 50\% ernjedrigt. Nacls $\Lambda$ bbildung 4 ist dic strenge Lincarität bis $7,5 \mathrm{mg} /$ $100 \mathrm{~m} / \mathrm{gegeben}, \mathrm{d}$. h. der tatsächliche Reaktionsendpunkt wird erreicht. Bci 10 und $15 \mathrm{mg} / 100 \mathrm{~m} /$ ist dies nur noch zu 70-80\% der Fäl, da dic urspeüngliche Menge an GDH beibehalten wurde. Die Meßgenauigkeit am AutoAnalyzer berülırt dies nicht, da bei hohen Glyccrinkonzentrationen auch gleich hohe Standards mitgemessen werden. Bei Ërhöhung der Enæymmenge wird auch hier der lindpunkt erreicht.

Dic angegebenen $600 \mathrm{~m} /$ Renktionsgemisch genügen zur Messung von 300 Blutproben von je $100 \mu \mathrm{l}$, wolxei jede Messung ohne Personal und Erstanschatfung 0,60 DM, bei Erhohhung der Ën\%ymmenge 0,75 DM kostet. Im Vergleich claru betragen die Kosten einer Glycerinhestimmung mit der Bochringcr-'I'cstpackung oder mit der manuellen Muthode nach IV(tiLANI) mit $200 \mu l$ Serum ctwa 1,20 1)M.

Der mit dem beschrichenen Reaktionsgemisch meßbare Koneentrationsbereich des Glycurins betrïgt bei $100 \mu$ l Blut. $0,2-15 \mathrm{mg} / 100 \mathrm{~m} /$, mit $200 \mu /$ Blut können noch $0,1 \mathrm{mg} / 100 \mathrm{~m} / \mathrm{gemessen}$ werden. Die von ligosinis und Kkliu's (24) angegebene untere Meßgren\%c licgt 
bei $0,04 \mathrm{mg} / 100 \mathrm{ml}$ (bei $200 \mu l$ Serum). Dazu ist eine exakte Ablesung von $\mathrm{E}=0,005$ nötig.

Dies gelang uns jedoch bei vorangegangenen manuellen Glycerinbestimmungen nach EgGSTEIN und KREUTZ (24) und Verwendung von Serum an Stelle von Glycerinlösungen wegen des stets auftretenden Reaktionsdrifts nicht. Die angegebene Umsatzrate von $20-40$ Doppelbestimmungen pro Stunde pro Mitarbeiter konnten wir ebenfalls nicht erreichen, da die Warte- zeiten bị zum Reaktionsstillstand vor Zugabe von GK meist länger als 10 Minuten waren.

Fräulèin J. MESțE danken wir für ihre ausgezeichnețe Mitarbeit.

\section{Anmerkung bei der Korrektur}

Infolge eines New Yorker Hafenarbeiterstreiks wurde uns erst nach Einsendung des Manusktipts eine Publikation mit gleichem Thema von H. Ko und M. E. Royer, Analyt. Biochem. 26, 18 (1968), bekannt. Wir glauben, daß unser Verfahren im Vergleich zu dem von Ko und ROYER angegebenen wesentliche Vorteile aufweist.

\section{Literatur}

1. Eggstern, M., Wien klin. Wschr. 79, 709 (1967). - 2. STernBERG, D., in Biochemical Society Symposia 24 (1963), "The control of Lipid Metabolism", S. 111. - 3. LAMBERT, M. und A. C. Neish, Canad. J. Res. 28, 83 (1950). - 4. Harvey, S. C. und V. HighBy, Arch. Biochem. Biophysics 30, 14 (1951). - 5. Korn, E. D., J. biol. Chemistry 215, 1 (1955). - 6. BAILEY, M., J. Laborat. clin. Med. S. Louis 54, 158 (1959). - 7. MENDELSOHN, O. und A. Antonis, J. Lipid Res. 2, 45 (1961). - 8. McAloren, J. T. und G. F. Reynolds, Analyt. clin. Acta 32, 170 (1965). 9. Laurell, S. und G. Tibbling, Clin. chim. Acta Amsterdam 13, 317 (1966). - 10. Pays, M., P. Malangeau und R. Bourdon, Ann. pharmaceut. franc. 15, 29 (1967). - 11. Freitas, A. S. W. de, Canad. J. Biochem. 45, 1041 (1967). - 12. Wreland, O., Biochem. Z. 329, 313 (1957). - 13. HAGEN, J. H. und P. B. HAGEN, Canad.
J. Biochem. 40, 1129 (1962). - 14. Kreutz, F. H., Klin. Wschr. 40, 362 (1962). - 15. Garland, P. B. und P. J. RandLE, Nature London 196, 987 (1962). - 16. Frings, C. S. und H. L. Pardue, Analyt. clin. Acta, 34, 228 (1966). - 17. Spinella, C. J. und M. Mager, J. Lipid Res. 7, 167 (1966). - 18. Pinter, J. K., J. A. Hayashi und J. A. Watson, Arch. Biochem. Biophysics 121, 404 (1967). - 19. Fain, J. N., N. Reed und R. Sapersterin, J. biol. Chemistry 242, 1887 (1967). - 20. KessLER, G. und H. Lederer in "Automation in Analytical Chemistry“, Technicon Symposia, 1965, S. 341. - 21. Lofland, H. B., Analyt. Biochem. 9, 393 (1964). - 22. Antonis, A., J. Amer. Oil Chemist's Soc. 44, 333 (1967). - 23. Eggstern, M., Klin. Wschr. 44, 267 (1966). 24. Eggstein, M. und F. H. Kreutz, Klin. Wschr. 44, 262 (1966).
Dr. Uwe Harding

Biochemische Forschungslaboratorien der Fa. Dr. Karl Thomae GmbH 795 Biberach an der Riss 

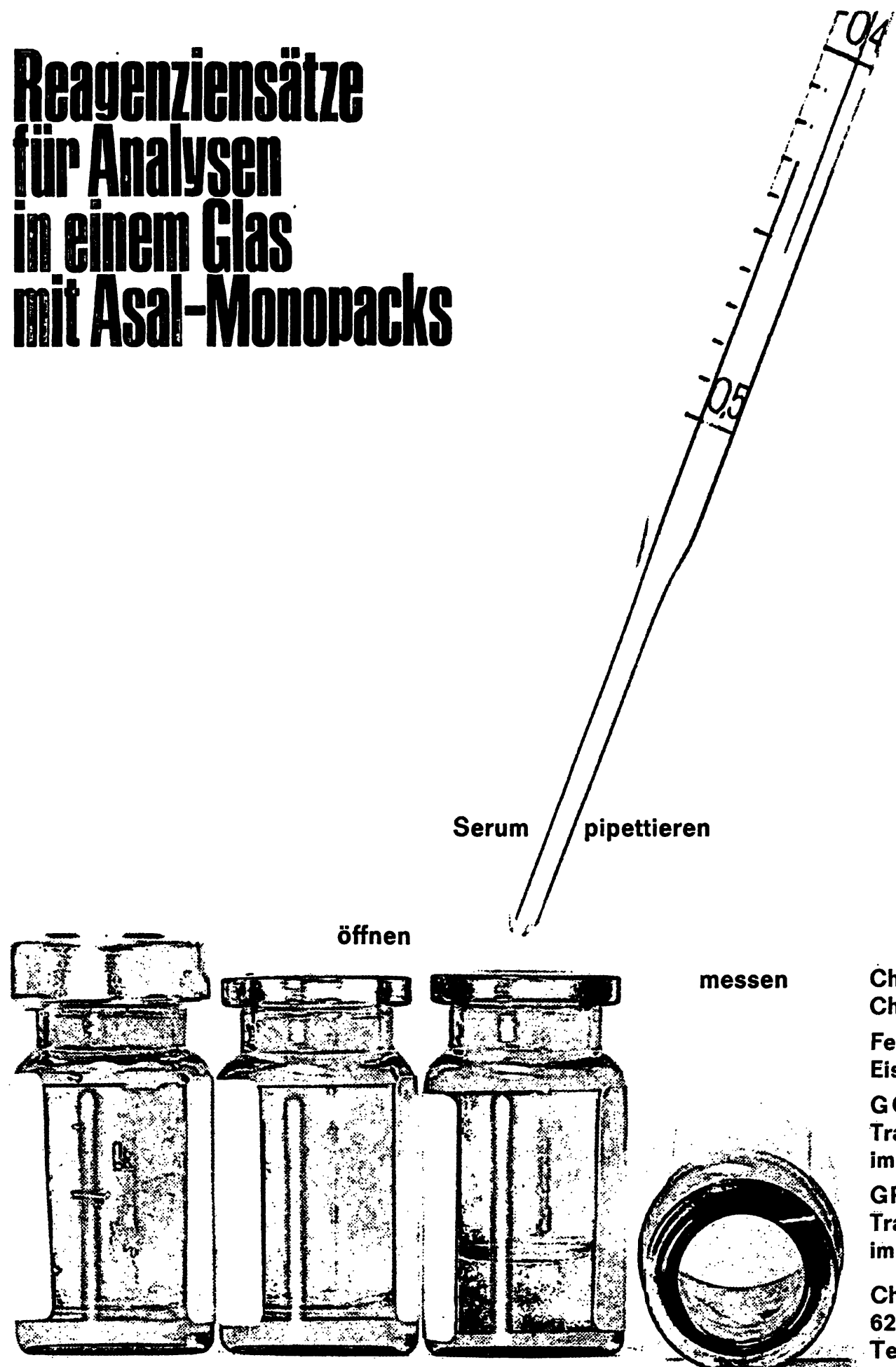

messen ChE-Monopack

Cholinesterase im Serum

Ferro-Monopack

Eisen im Serum

GOT-Monopack

Transaminaseaktivität im Serum

GPT-Monopack

Transaminaseaktivität im Serum

Chemische Fabrik Asal 6231 Schwalbach/Taunus Telefon (061 96) 3031 


\title{
Sigma freut sich, die kompletteste Liste
}

\section{cyclischer Nucleosidphosphate}

\author{
anbieten zu können.
}

Alle sind in den Sigma-Laboratorien hergestellt.

cycl. Adenosin-2',3'-monophosphorsäure

Ammonium- oder andere Salze, je nach Vorrat
$25 \mathrm{mg}-6,25 \quad 100 \mathrm{mg}-17,10 \quad 250 \mathrm{mg}-34,20 \quad 500 \mathrm{mg}-57,00 \quad 1 \mathrm{~g}-95,00$

cycl. Adenosin-3',5'-monophosphorsäure

$\begin{array}{ccccc}\text { kristallin, stimuliert unter anderem die Bildung von Phosphorylase } & \cdot \\ 25 \mathrm{mg}-4,90 \quad 50 \mathrm{mg}-8,50 \quad 100 \mathrm{mg}-14,50 \quad 250 \mathrm{mg}-30,00 \quad 500 \mathrm{mg}-54,00 & 1 \mathrm{~g}-93,50\end{array}$

cycl. Cytidin-2',3'-monophosphorsäure

$$
\begin{array}{cccc}
100 \mathrm{mg}-7,20 & 250 \mathrm{mg}-17,90 \begin{array}{c}
\text { Natriumsalz } 95-99 \% \\
500 \mathrm{mg}-29,70
\end{array} & 1 \mathrm{~g}-49,50 & 5 \mathrm{~g}-220,00 \\
\text { cycl. Cytidin-3',5'-monophosphorsäure } \\
\text { freie Säure, Reinheitsgrad I, etwa } 98 \% \\
5 \mathrm{mg}-10,00 \quad 25 \mathrm{mg}-33,00 \quad 100 \mathrm{mg}-90,00 \quad 500 \mathrm{mg}-300,00 & 1 \mathrm{~g}-500,00
\end{array}
$$

N6-2'0-Dibutyryl-adenosin-3',5'-monophosphorsäure, cycl.

$5 \mathrm{mg}-3,00 \quad 25 \mathrm{mg}-10,00 \quad 100 \mathrm{mg}-26,75 \quad 250 \mathrm{mg}-55,00$

cycl. Guanosin-2',3'-monophosphorsäure

$$
\begin{array}{cccc}
100 \mathrm{mg}-8,50 & 250 \mathrm{mg}-19,50 & 500 \mathrm{mg}-36,00 & 1 \mathrm{~g}-60,00 \\
\multicolumn{5}{c}{\text { Mono-tributylaminsalz, etwa } 98 \% \text {, wasserlöslich }} \\
25 \mathrm{mg}-5,75 \quad 100 \mathrm{mg}-16,20 \quad 250 \mathrm{mg}-32,40 & 1 \mathrm{~g}-90,00
\end{array}
$$

cycl. Guanosin-3',5'-monophosphorsäure

$$
\begin{array}{ccc}
5 \mathrm{mg} & \text { Reinheitsgrad II, etwa } 90 \% \text {, Ammoniumsalz } \\
25,00 & 25 \mathrm{mg}-24,00 \quad 100 \mathrm{mg}-67,50 & 1 \mathrm{~g}-350,00
\end{array}
$$

cycl. Thymidin-3',5'-monophosphorsäure

Reinheitsgrad I, etwa $98 \%$, Natrium- oder Ammoniumsalz
$25 \mathrm{mg}-24,50 \quad 100 \mathrm{mg}-67,50 \quad 500 \mathrm{mg}-225,00 \quad 1 \mathrm{~g}-375,00$

cycl. Uridin-2',3'-monophosphorsäure

$100 \mathrm{mg}-8,40 \quad 500 \mathrm{mg}-30,00 \quad 1 \mathrm{~g}-50,00$

cycl. Uridin-3',5'-monophosphorsäure

$\begin{array}{llll} & \text { Reinheitsgrad I, etwa } 98 \% \text {, Ammoniumsalz } & \\ \mathrm{mg}-5,00 & 25 \mathrm{mg}-16,50 \quad 100 \mathrm{mg}-45,00 \quad 500 \mathrm{mg}-150,00 & 1 \mathrm{~g}-250,00\end{array}$

Sämtliche Preise sind in US-Dollars angegeben.

Sigma-Reagenzien sind in der ganzen Welt durch den Fachhandel oder direkt aus St. Louis beziehbar.

Telegramme: SIGMACHEM, St. Louis, Missouri

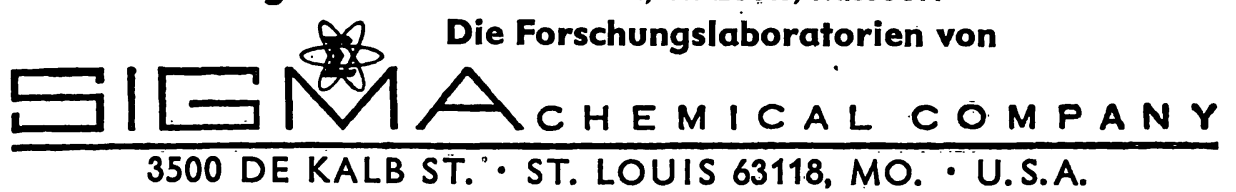

\section{MANUFACTURERS OF THE, FINEST BIOCHEMICALS AVAILABLE}

